\title{
Immersion and Invariance Based Nonlinear Adaptive Flight Control
}

\author{
L. Sonneveldt, E. R. van Oort $\dagger$ Q. P. Chu $\ddagger$ and J. A. Mulder ${ }^{\ddagger}$ \\ Delft University of Technology, P.O. Box 5058, 2600 GB Delft, The Netherlands
}

\begin{abstract}
In this paper a theoretical framework for nonlinear adaptive flight control is developed and applied to a simplified, over-actuated nonlinear fighter aircraft model. The framework is based on a modular adaptive backstepping scheme with a new type of nonlinear estimator. The nonlinear estimator is constructed using an invariant manifold based approach which allows for prescribed dynamics to be assigned to the estimation error. Attractivity of the manifold is ensured with the addition of dynamic scaling factors and output filters to the design procedure. The properties of the estimator can be exploited by designing a command filtered backstepping control law that renders the closed-loop system input-tostate stable with respect to the parameter estimation error. It is demonstrated that the resulting modular adaptive controller is easier to tune compared to controllers obtained using the classical adaptive backstepping approaches. Furthermore, the performance of the adaptive controller does not suffer from unpredictable dynamical behavior of the parameter update laws. This is illustrated in numerical simulations where several types of realistic failures are introduced in the aircraft model.
\end{abstract}

\section{Nomenclature}

$\begin{array}{ll}g_{0} & \text { gravity constant, } \mathrm{ft} / \mathrm{s}^{2} \\ i_{1}, i_{2}, i_{3} & \text { non-dimensional inertia parameters, }- \\ p, q, r & \text { body-axis roll, pitch and yaw rates, rad } / \mathrm{s} \\ V & \text { total velocity, ft/s } \\ \alpha & \text { angle of attack, rad } \\ \alpha_{0} & \text { trimmed angle of attack, rad } \\ \beta & \text { angle of sideslip, rad } \\ \delta_{*} & \text { deflection of control surface }{ }^{*}, \mathrm{rad} \\ \phi & \text { roll angle, rad } \\ \theta & \text { pitch angle, rad } \\ \theta_{0} & \text { trimmed pitch angle, rad }\end{array}$

\section{Introduction}

In the past two decades a considerable amount of literature has been devoted to nonlinear adaptive control design methods for a variety of flight control problems where parametric uncertainties in the system dynamics are involved. ${ }^{1-9}$ Recursive, Lyapunov-based adaptive backstepping ${ }^{10,11}$ is among the most widely studied of these methods. The main attractions of adaptive backstepping based control laws lie in their provable convergence and stability properties as well as in the fact that the they can be applied to a broad class of nonlinear systems.

*PhD. Student, Aerospace Software and Technology Institute, l.sonneveldt@tudelft.nl.

${ }^{\dagger}$ PhD. Student, Aerospace Software and Technology Institute, e.r.vanoort@tudelft.nl.

${ }^{\ddagger}$ Associate Professor, Control \& Simulation Division, q.p.chu@tudelft.nl.

§Professor, Control \& Simulation Division, j.a.mulder@tudelft.nl. 
However, despite a number of refinements over the years, the adaptive backstepping method also has a number of shortcomings. The most important of these is that the parameter estimation error is only guaranteed to be bounded and converging to an unknown constant value, yet little can be said about its dynamical behavior. Unexpected dynamical behavior of the parameter update laws may lead to an undesired transient response of the closed-loop system. Furthermore, increasing the adaptation gain will lead to faster parameter convergence, but will not necessarily improve the response of the system. This makes it difficult to properly tune an integrated adaptive backstepping controller for large and complex systems, as is demonstrated for a high-fidelity F-16 model in Ref. 12.

One solution to this problem is to introduce a modular input-to-state stable backstepping approach ${ }^{13}$ with a separate identifier that is not of the Lyapunov-type, e.g. the well known recursive least-squares identifier. ${ }^{14}$ Since the certainty equivalence principle does not hold, in general, for nonlinear systems, the control law has to be robustified against the time-varying character of the parameter estimates. However, the nonlinear damping terms introduced to achieve this robustness can lead to undesirable high gain control. Furthermore, the controller loses some of the strong stability properties with respect to the integrated adaptive backstepping approach.

In Refs. 15-17, a different class of Lyapunov-based adaptive controllers has been developed based on the immersion and invariance (I\&I) methodology. This approach allows for prescribed stable dynamics to be assigned to the parameter estimation error, thus leading to a modular control scheme which is much easier to tune than an adaptive backstepping controller. However, this shaping of the dynamics relies on the solution of a partial differential matrix inequality, which is difficult to solve for multivariable systems. This limitation is removed in Ref. 18 using a dynamic extension consisting of output filters and dynamic scaling factors added to the estimator dynamics.

In this study, the approach of Ref. 18 is used to derive a nonlinear I\&I based estimator, which in combination with a command filtered backstepping feedback controller ${ }^{19,20}$ results in a nonlinear adaptive control framework with guaranteed global asymptotic stability of the closed-loop system. As an example, the flight control design for a simplified, over-actuated fighter aircraft model is considered. It is assumed that the stability and control derivatives of the model are not exactly known and that they can change during flight due to structural damage or control surface failures. The performance of the proposed adaptive flight controller is verified in numerical simulations of various maneuvers involving several cases of control surface runaways (hard-overs).

The paper is outlined as follows. First, concept of immersion and invariance is explained and illustrated with a simple example in Section II. After that, the theory behind the immersion and invariance based nonlinear adaptive estimator and the backstepping feedback controller are discussed in Section III. The dynamics of the fighter aircraft model are introduced in Section IV, after which the adaptive flight control law for the aircraft model is constructed in Section V. Section VI validates the performance of the adaptive control law using numerical simulations performed in MATLAB/Simulink ${ }^{\circledR}$. Finally, a summary of the results and the conclusions are given in Section VII.

\section{The Immersion and Invariance Concept}

Immersion and invariance is a relatively new approach to designing nonlinear controllers or estimators for (uncertain) nonlinear systems. ${ }^{21,22}$ As the name suggests, the method relies on the notions of system immersion and manifold invariance, but used from another perspective. The idea behind the I\&I approach is to capture the desired behavior of the system to be controlled with a target dynamical system. This way the control problem is reduced to the design of a control law which guarantees that the controlled system asymptotically behaves like the target system.

The I\&I method is applicable to a variety of control problems, but it is easiest to illustrate the approach with a basic stabilization problem of an equilibrium point of a nonlinear system. Consider the general system

$$
\dot{x}=f(x)+g(x) u,
$$

where $x \in \mathbb{R}^{n}$ and $u \in \mathbb{R}^{m}$. The control problem is to find a state feedback control law $u=v(x)$ such that the closed-loop system has a globally asymptotic stable equilibrium at the origin. The first step of the I\&I approach is to find a target dynamical system

$$
\dot{\xi}=\alpha(x),
$$


where $\xi \in \mathbb{R}^{p}, p<n$, which has a globally asymptotically stable equilibrium at the origin, a smooth mapping $x=\pi(\xi)$, and a control law $v(x)$ such that

$$
f(\pi(\xi))+g(\pi(\xi)) v(\pi(\xi))=\frac{\partial \pi}{\partial \xi} \alpha(\xi)
$$

If these conditions hold, then any trajectory $x(t)$ of the closed-loop system

$$
\dot{x}=f(x)+g(x) v(x)
$$

is the image through the mapping $\pi(\xi)$ of a trajectory $\xi(t)$ of the target system Eq. (2). Note that the rank of $\pi$ is equal to the dimension of $\xi$. The second step is to find a control law that renders the manifold $x=\pi(\xi)$ attractive and keeps the closed-loop trajectories bounded. This way the closed-loop system will asymptotically behave like the desired target system and hence stability is ensured.

From the above discussion, it follows that the control problem has been transformed into the problem of the selection of a target dynamical system. This is, in general, a non-trivial task, since the solvability of the underlying control design problem depends on this selection. However, in many cases of practical interest it is possible to identify natural target dynamics. Examples of different applications are given in Ref. 22.

In this paper, the focus lies on adaptive control, hence the I\&I approach is used to develop a framework for adaptive stabilization of nonlinear systems with parametric uncertainties. Consider again the system Eq. (1) with an equilibrium $x_{e}$ to be stabilized, but where the functions $f(x)$ and $g(x)$ now depend on an unknown parameter vector $\theta \in \mathbb{R}^{q}$. The goal is to find an adaptive state feedback control law of the form

$$
\begin{aligned}
u & =v(x, \hat{\theta}) \\
\dot{\hat{\theta}} & =w(x, \hat{\theta}),
\end{aligned}
$$

such that all trajectories of the closed-loop system Eqs. (1), (5) are bounded and $\lim _{t \rightarrow \infty} x=x_{e}$. To this end it is assumed that a full-information control law $v(x, \theta)$ exists. The I\&I adaptive control problem is then defined as follows. ${ }^{21}$

Definition II.1. The system (1) is said to be adaptively IEI stabilizable if there exist functions $\beta(x)$ and $w(x)$ such that all trajectories of the extended system

$$
\begin{aligned}
\dot{x} & =f(x)+g(x) v(x, \hat{\theta}+\beta(x)) \\
\dot{\hat{\theta}} & =w(x, \hat{\theta})
\end{aligned}
$$

are bounded and satisfy

$$
\lim _{t \rightarrow \infty}[g(x(t)) v(x(t), \hat{\theta}(t)+\beta(x(t)))-g(x(t)) v(x(t), \theta)]=0 .
$$

It is not difficult to see that for all trajectories staying on the manifold

$$
\mathcal{M}=\left\{(x, \hat{\theta}) \in \mathbb{R}^{n} \times \mathbb{R}^{q} \mid \hat{\theta}-\theta+\beta(x)=0\right\}
$$

condition (7) holds. Moreover, by Definition II.1, adaptive I\&I stabilizability implies that

$$
\lim _{t \rightarrow \infty} x=x_{e}
$$

Note that the adaptive control framework designed with the I\&I approach is not of the certainty equivalence type in the strict sense due to the $\beta(x)$ term, i.e. the parameter estimate is not used directly by the static feedback controller. Furthermore, note that, in general, $f(x)$ and $g(x)$ depend on the unknown $\theta$ and therefore the parameter estimate $\hat{\theta}$ does not necessarily converge to the true parameter values. However, in many cases it is also possible to establish global stability of the equilibrium $(x, \hat{\theta})=\left(x_{e}, \theta\right)$. This is illustrated in the following simple example. 


\section{Example II.1}

Consider the feedback linearizable system

$$
\dot{x}=\theta x^{3}+x+u,
$$

where $\theta \in \mathbb{R}$ is an unknown constant parameter. If $\theta$ were known, the equilibrium point $x=0$ would be globally asymptotically stabilized by the control law

$$
u=-\theta x^{3}-c x, \quad c>1 .
$$

Since $\theta$ is not known, $\theta$ is replaced by its estimate $\hat{\theta}$ in the certainty equivalence controller

$$
\begin{aligned}
u & =-\hat{\theta} x^{3}-(c-1) x, \\
\dot{\hat{\theta}} & =w,
\end{aligned}
$$

where $w$ is the parameter update law. As before, the control Lyapunov function is selected as

$$
V(x, \hat{\theta})=\frac{1}{2} x^{2}+\frac{1}{2 \gamma}(\theta-\hat{\theta})^{2},
$$

with $\gamma>0$. Selecting the update law

$$
w=\gamma x^{4}
$$

renders the derivative of $V$ equal to

$$
\dot{V}=-(c-1) x^{2} .
$$

Hence, the equilibrium $(x, \tilde{\theta})=0$ is globally stable and from Barbalat's lemma 23 it follows that $\lim _{t \rightarrow \infty} x=$ 0 . However, no conclusions can be drawn about the behavior of the parameter estimation error $\theta-\hat{\theta}$, except that it converges to a constant value. The dynamical behavior of the estimation error may be unacceptable in terms of transient response of the closed-loop system.

Alternatively, the adaptive control problem can be placed in the I\&I framework by considering the augmented system

$$
\begin{aligned}
\dot{x} & =\theta x^{3}+x+u \\
\dot{\hat{\theta}} & =w
\end{aligned}
$$

and by defining the one-dimensional manifold

$$
\mathcal{M}=\left\{(x, \hat{\theta}) \in \mathbb{R}^{2} \mid \hat{\theta}-\theta+\beta(x)=0\right\}
$$

in the extended space $(x, \hat{\theta})$, where $\beta(x)$ is a continuous function yet to be specified. If the manifold $\mathcal{M}$ is invariant, the dynamics of the x-subsystem of Eq. (14) restricted to this manifold can be written as

$$
\dot{x}=(\hat{\theta}+\beta(x)) x^{3}+x+u .
$$

Hence, the dynamics of the system are completely known and the equilibrium $x=0$ can be asymptotically stabilized by the control law

$$
u=-c x-(\hat{\theta}+\beta(x)) x^{3}, \quad c>1 .
$$

To render this design feasible, the first step of the I\&I approach consists of finding an update law $w$ that renders the manifold $\mathcal{M}$ invariant. To this end, consider the dynamics of the 'off-the-manifold' coordinate, i.e. the estimation error

$$
\sigma \triangleq \hat{\theta}-\theta+\beta(x)
$$

which are given by

$$
\begin{gathered}
\dot{\sigma}=w+\frac{\partial \beta}{\partial x}\left((\hat{\theta}+\beta(x)-\sigma) x^{3}+x+u\right) . \\
4 \text { of } 18
\end{gathered}
$$


If the update law $w$ is selected as

$$
w=-\frac{\partial \beta}{\partial x}\left((\hat{\theta}+\beta(x)) x^{3}+x+u\right)=\frac{\partial \beta}{\partial x}(c-1) x
$$

the manifold $\mathcal{M}$ is invariant and the off-the-manifold dynamics are described by

$$
\dot{\sigma}=-\frac{\partial \beta}{\partial x} x^{3} \sigma .
$$

Consider the Lyapunov function $V=\frac{1}{2} \sigma^{2}$ whose time derivative along the trajectories of (20) satisfies

$$
\dot{V}=-\frac{\partial \beta}{\partial x} x^{3} \sigma^{2}
$$

where $\gamma>0$. To render this expression negative definite, one possible choice for the function $\beta(x)$ is given as

$$
\beta(x)=\gamma \frac{x^{2}}{2} .
$$

An alternative solution with dead-zones is given as

$$
\beta(x)=\left\{\begin{array}{lll}
\frac{\gamma}{2}\left(x-\eta_{0}\right)^{2} & \text { if } & x>\eta_{0} \\
\frac{\gamma}{2}\left(x+\eta_{0}\right)^{2} & \text { if } & x<-\eta_{0} \\
0 & \text { if } & |x| \leq \eta_{0}
\end{array},\right.
$$

with $\eta_{0}>0$ the dead-zone constant. It can be concluded that the system Eq. (20) has a globally stable equilibrium at zero and $\lim _{t \rightarrow \infty} x^{3} \sigma=0$. The resulting closed-loop can be written in $(x, \sigma)$-coordinates as

$$
\begin{aligned}
\dot{x} & =-(c-1) x-x^{3} \sigma \\
\dot{\sigma} & =-\gamma x^{4} \sigma
\end{aligned}
$$

which has a global stable equilibrium at the origin and $x$ converges to zero. Moreover, the extra $\beta(x) x^{3}$ term in the control law Eq. (16) renders the closed-loop system input-to-state stable with respect to the parameter estimation error $\theta-\hat{\theta}$.

The response of the closed-loop system with the I\&I adaptive controller is compared to the response of the system with the standard adaptive controller designed at the beginning of this example. The tuning parameters of both designs are selected the same. The real $\theta$ is equal to 2 , but the initial parameter estimate is 0 . As can be seen in Figure 1, both controllers manage to regulate the state to zero. Note that it is not guaranteed that the estimate of the I\&I design converges to the true value, only that $\lim _{t \rightarrow \infty} x^{3} \sigma=0$.

The closed-loop system Eq. (24) can be regarded as a cascaded interconnection between two stable systems which can be tuned via the constants $c$ and $\gamma$. This modularity makes the I\&I adaptive controller much easier to tune than the standard adaptive design. As a result, the performance of the adaptive system can be significantly improved.

\section{Immersion and Invariance Based Nonlinear Adaptive Control}

Extending the I\&I approach outlined in the last section to higher-order nonlinear systems with unmatched uncertainties is by no means straight-forward. In Ref. 15 an I\&I based estimator is designed for a class of lower-triangular nonlinear systems. The estimator allows for prescribed dynamics to be assigned to the parameter estimation error. However, the shaping of the dynamics relies on the solution of a partial differential matrix inequality, which is, in general, very difficult to solve for most physical systems. This limitation of the estimator design was removed in Ref. 18 with the introduction of a dynamic scaling factor in the estimator dynamics and by adding an output filter to the design. Dynamic scaling has been widely used in the design of high-gain observers, see e.g. Ref. 24. In this section an I\&I estimator with dynamic 

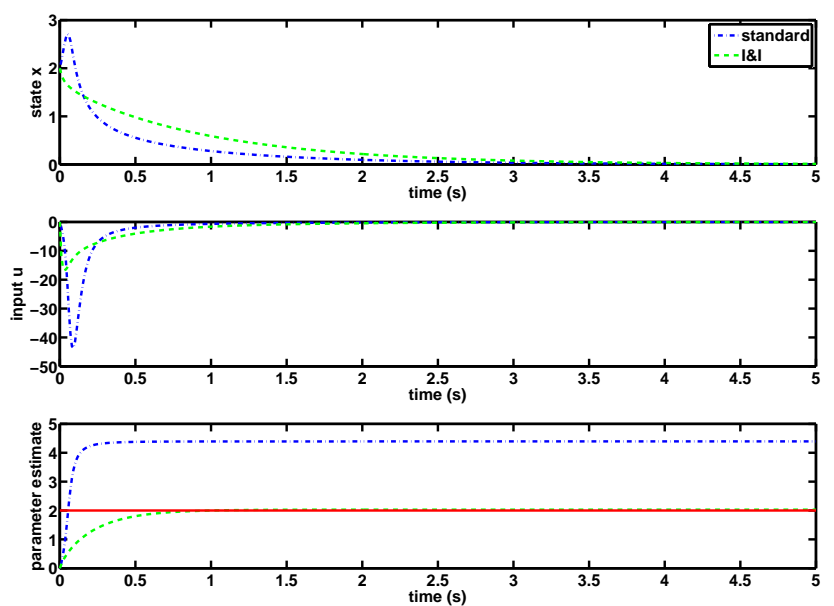

Figure 1. State $x$, control effort $u$ and parameter estimate $\hat{\theta}$ for initial values $x(0)=2, \hat{\theta}(0)=0$, control gain $c=2$ and update gain $\gamma=1$ for the closed-loop system with standard adaptive design and with the I\&I adaptive design.

scaling and output filter is combined with a command filtered adaptive backstepping control design approach to arrive at a modular adaptive control framework.

Consider the class of linearly parametrized systems of the form

$$
\dot{x}_{i}=x_{i+1}+\varphi_{i}(x)^{T} \theta_{i}, \quad i=1, \ldots, n,
$$

with states $x_{i} \in \mathbb{R}, i=i, \ldots, n$ and control input $u \in \mathbb{R}$. Note that for notational convenience $x_{n+1}=u$. The functions $\varphi_{i}(x, u)$ are the known, smooth regressors and $\theta_{i} \in \mathbb{R}^{p_{i}}$ are vectors of unknown constant parameters. The control objective is to track a smooth reference signal $x_{1, r}$, for which the first derivative is known and bounded, with the state $x_{1}$. The adaptive control design is done in two steps. First, an overparametrized estimator for the unknown parameter vector $\theta$ is designed. In the second step a controller is designed that ensures that $\lim _{t \rightarrow \infty} x_{1}=y_{r}$ and all other states are bounded.

\section{III.A. Estimator Design with Dynamic Scaling}

The construction of an estimator for $\theta_{i}$ starts by defining the scaled estimation errors as

$$
\sigma_{i}=\frac{\hat{\theta}_{i}-\theta_{i}+\beta_{i}\left(x_{i}, \hat{x}\right)}{r_{i}}, \quad i=1, \ldots, n,
$$

where $r_{i}$ are scalar dynamic scaling factors, $\hat{\theta}_{i}$ are the estimator states and $\beta_{i}\left(x_{i}, \hat{x}\right)$ continuously differentiable vector functions yet to be specified. Let $e_{i}=\hat{x}_{i}-x_{i}$, then the filtered states $\hat{x}_{i}$ are obtained from

$$
\dot{\hat{x}}_{i}=x_{i+1}+f_{i}(x)+\varphi_{i}(x)^{T}\left(\hat{\theta}_{i}+\beta_{i}\left(x_{i}, \hat{x}\right)\right)-k_{i}(x, r, e) e_{i},
$$

where $k_{i}(x, r, e)$ are positive functions. Using the above definitions, the dynamics of $\sigma_{i}$ are given by

$$
\begin{aligned}
\dot{\sigma}_{i} & =\frac{1}{r_{i}}\left(\dot{\hat{\theta}}_{i}+\frac{\partial \beta_{i}}{\partial x_{i}}\left(x_{i+1}+\varphi_{i}(x)^{T} \theta_{i}\right)+\sum_{j=1}^{n} \frac{\partial \beta_{i}}{\partial \hat{x}_{j}} \dot{\hat{x}}_{j}\right)-\frac{\dot{r}_{i}}{r_{i}} \sigma_{i} \\
& =\frac{1}{r_{i}}\left(\dot{\hat{\theta}}_{i}+\frac{\partial \beta_{i}}{\partial x_{i}}\left[x_{i+1}+\varphi_{i}(x)^{T}\left(\hat{\theta}_{i}+\beta_{i}\left(x_{i}, \hat{x}\right)-r_{i} \sigma_{i}\right)\right]+\sum_{j=1}^{n} \frac{\partial \beta_{i}}{\partial \hat{x}_{j}} \dot{\hat{x}}_{j}\right)-\frac{\dot{r}_{i}}{r_{i}} \sigma_{i} .
\end{aligned}
$$

By selecting the update laws for $\dot{\hat{\theta}}_{i}$ as

$$
\dot{\hat{\theta}}_{i}=-\frac{\partial \beta_{i}}{\partial x_{i}}\left(x_{i+1}+\varphi_{i}(x)^{T}\left(\hat{\theta}_{i}+\beta_{i}\left(x_{i}, \hat{x}\right)\right)\right)-\sum_{j=1}^{n} \frac{\partial \beta_{i}}{\partial \hat{x}_{j}} \dot{\hat{x}}_{j},
$$


the dynamics of $\sigma_{i}$ are reduced to

$$
\dot{\sigma}_{i}=-\frac{\partial \beta_{i}}{\partial x_{i}} \varphi_{i}(x)^{T} \sigma_{i}-\frac{\dot{r}_{i}}{r_{i}} \sigma_{i}=-\left(\frac{\partial \beta_{i}}{\partial x_{i}} \varphi_{i}(x)^{T}+\frac{\dot{r}_{i}}{r_{i}}\right) \sigma_{i} .
$$

The system Eq. (29) can be seen as a linear time varying system with a block diagonal dynamic matrix. Hence, the problem of designing an estimator $\hat{\theta}_{i}$ is now reduced to the problem of finding functions $\beta_{i}$ such that the diagonal blocks are rendered negative semi-definite. In Ref. 18 the functions $\beta_{i}$ are selected as

$$
\beta_{i}\left(x_{i}, \hat{x}\right)=\gamma_{i} \int_{0}^{x_{i}} \varphi_{i}\left(\hat{x}_{1}, \ldots, \hat{x}_{i-1}, \chi, \hat{x}_{i+1}, \ldots, \hat{x}_{n}\right) d \chi
$$

where $\gamma_{i}>0$. Since the regressors $\varphi_{i}(x)$ are continuously differentiable, the expression

$$
\sum_{j=1}^{n} e_{j} \delta_{i j}(x, e)=\varphi_{i}(x)-\varphi_{i}\left(\hat{x}_{1}, \ldots, \hat{x}_{i-1}, x_{i}, \hat{x}_{i+1}, \ldots, \hat{x}_{n}\right), \quad \delta_{i i} \equiv 0,
$$

holds for some functions $\delta_{i j}(x, e)$. Substituting Eqs. $(30,31)$ into Eq. (29) yields the $\sigma_{i}$-dynamics

$$
\dot{\sigma}_{i}=-\gamma_{i} \varphi_{i}(x) \varphi_{i}(x)^{T} \sigma_{i}+\gamma_{i} \sum_{j=1}^{n} e_{j} \delta_{i j}(x, e) \varphi_{i}(x)^{T} \sigma_{i}-\frac{\dot{r}_{i}}{r_{i}} \sigma_{i} .
$$

Furthermore, from Eqs. (25) and (27), the dynamics of $e_{i}=\hat{x}_{i}-x_{i}$ are given by

$$
\dot{e}_{i}=-k_{i}(x, r, e) e_{i}+r_{i} \varphi_{i}(x)^{T} \sigma_{i} .
$$

The system consisting of Eqs. (32) and (33) has an equilibrium at zero, which can be rendered globally uniformly stable by selecting the dynamics of the scaling factors $r_{i}$ and the functions $k_{i}(x, r, e)$ as defined in the following lemma. ${ }^{18}$

Lemma III.1. Consider the system (25) and let

$$
\dot{r}_{i}=c_{i} r_{i} \sum_{j=1}^{n} e_{j}^{2}\left|\delta_{i j}(x, e)\right|^{2}, \quad r_{i}(0)=1,
$$

with $c_{i} \geq \gamma_{i} n / 2$, where $|$.$| denotes the 2-norm, and$

$$
k_{i}(x, r, e)=\lambda_{i} r_{i}^{2}+\epsilon \sum_{j=1}^{n} c_{j} r_{j}^{2}\left|\delta_{j i}(x, e)\right|^{2}
$$

where $\lambda_{i}>0$ and epsilon $>0$ are constants. Then the system consisting of Eqs. (32), (33) and (34) has a globally uniformly stable manifold of equilibria defined by $\mathcal{M}=\{(\sigma, r, e) \mid \sigma=e=0\}$. Moreover, $\sigma_{i}(t) \in \mathcal{L}_{\infty}$, $r_{i}(t) \in \mathcal{L}_{\infty}, e_{i}(t) \in \mathcal{L}_{2} \cap \mathcal{L}_{\infty}$ and $\varphi_{i}(x(t))^{T} \sigma_{i}(t) \in \mathcal{L}_{2}$ for all $i=1, \ldots, n$. If, in addition, $\varphi_{i}(x(t))$ and its time derivative are bounded, it follows that $\lim _{t \rightarrow \infty} \varphi_{i}(x(t))^{T} \sigma_{i}(t)=0$.

Proof: Consider the Lyapunov function $V_{i}\left(\sigma_{i}\right)=\frac{1}{2 \gamma_{i}} \sigma_{i}^{T} \sigma_{i}$. Taking the time derivative of $V_{i}$ along the trajectories of Eq. (32) results in

$$
\begin{aligned}
\dot{V}_{i} & =-\left(\varphi_{i}^{T} \sigma_{i}\right)^{2}+\sum_{j=1}^{n} e_{j} \sigma_{i}^{T} \delta_{i j} \varphi_{i}^{T} \sigma_{i}-\frac{\dot{r}_{i}}{\gamma_{i} r_{i}}\left|\sigma_{i}\right|^{2} \\
& =-\left(\varphi_{i}^{T} \sigma_{i}\right)^{2}+\sum_{j=1}^{n}\left[\frac{1}{2 n}\left(\varphi_{i}^{T} \sigma_{i}\right)^{2}+\frac{n}{2} e_{j}^{2}\left(\delta_{i j}^{T} \sigma_{i}\right)^{2}\right]-\sum_{j=1}^{n}\left[\frac{1}{\sqrt{2 n}} \varphi_{i}^{T} \sigma_{i}-\sqrt{\frac{n}{2}} e_{j} \delta_{i j}^{T} \sigma_{i}\right]^{2}-\frac{\dot{r}_{i}}{\gamma_{i} r_{i}}\left|\sigma_{i}\right|^{2} \\
& \leq-\frac{1}{2}\left(\varphi_{i}^{T} \sigma_{i}\right)^{2}+\frac{n}{2} \sum_{j=1}^{n} e_{j}^{2}\left(\delta_{i j}^{T} \sigma_{i}\right)^{2}-\frac{\dot{r}_{i}}{\gamma_{i} r_{i}}\left|\sigma_{i}\right|^{2} .
\end{aligned}
$$


Substituting the dynamic scaling terms $r_{i}$ as given by Eq. (34) and by applying the inequality $\left|\delta_{i j}^{T} \sigma_{i}\right|<$ $\left|\delta_{i j}\right|\left|\sigma_{i}\right|$, the remaining indefinite term can be canceled such that

$$
\dot{V}_{i} \leq-\frac{1}{2}\left(\varphi_{i}^{T} \sigma_{i}\right)^{2}<0, \quad \sigma_{i} \neq 0 .
$$

Hence, the system Eq. (32) has a globally uniformly stable equilibrium at the origin, $\sigma_{i}(t) \in \mathcal{L}_{\infty}$ and $\varphi_{i}(x(t))^{T} \sigma_{i}(t) \in \mathcal{L}_{2}$ for all $i=1, \ldots, n$. If $\varphi_{i}(x(t))$ and its time derivative are bounded, it follows from Barbalat's lemma that $\lim _{t \rightarrow \infty} \varphi_{i}(x(t))^{T} \sigma_{i}(t)=0$. This implies that an asymptotic estimate of each parametric uncertainty term $\varphi_{i}(x)^{T} \theta_{i}$ in Eq. (25) is given by the term $\varphi_{i}(x)^{T}\left(\hat{\theta}_{i}+\beta_{i}\left(x_{i}, \hat{x}\right)\right)$.

The next design step is to select the positive functions $k_{i}(x, r, e)$ in such a way that the dynamics of $e_{i}$, given by Eq. (33), become globally asymptotically stable. Taking the time derivative of the augmented Lyapunov function $W_{i}\left(\sigma_{i}, e_{i}\right)=\frac{1}{2} e_{i}^{2}+\frac{1}{\lambda_{i}} V_{i}$, with constant $\lambda_{i}>0$, results in

$$
\begin{aligned}
\dot{W}_{i} & \leq-k_{i} e_{i}^{2}+r_{i} \varphi_{i}^{T} \sigma_{i} e_{i}-\frac{1}{2 \lambda_{i}}\left(\varphi_{i}^{T} \sigma_{i}\right)^{2} \\
& =-k_{i} e_{i}^{2}+\frac{\lambda_{i}}{2} r_{i}^{2} e_{i}^{2}+\frac{1}{2 \lambda_{i}}\left(\varphi_{i}^{T} \sigma_{i}\right)^{2}-\left[\sqrt{\frac{\lambda_{i}}{2}} r_{i} e_{i}-\frac{1}{\sqrt{2 \lambda_{i}}} \varphi_{i}^{T} \sigma_{i}\right]^{2}-\frac{1}{2 \lambda_{i}}\left(\varphi_{i}^{T} \sigma_{i}\right)^{2} \\
& \leq-\left[k_{i}-\frac{\lambda_{i}}{2} r_{i}^{2}\right] e_{i}^{2} .
\end{aligned}
$$

It is clear that selecting $k_{i}(x, r, e)>\frac{\lambda_{i}}{2} r_{i}^{2}$ renders the above expression negative definite, thus the equilibrium $\left(\sigma_{i}, e_{i}\right)=(0,0)$ is globally uniformly stable and $e_{i}(t) \in \mathcal{L}_{2} \cap \mathcal{L}_{\infty}$.

A final design step has to be made to ensure that the dynamic scalings $r_{i}$ remain bounded. Consider the Lyapunov function $V_{e}(e, \sigma, r)=\sum_{i=1}^{n}\left[W_{i}\left(\sigma_{i}, e_{i}\right)+\frac{\epsilon}{2} r_{i}^{2}\right]$ with $\epsilon>0$, for which the time derivative is given by

$$
\dot{V}_{e} \leq-\sum_{i=1}^{n}\left[k_{i}(x, r, e)-\frac{\lambda_{i}}{2} r_{i}^{2}\right] e_{i}^{2}+\epsilon \sum_{i=1}^{n}\left[c_{i} r_{i}^{2} \sum_{j=1}^{n} e_{j}^{2}\left|\delta_{i j}(x, e)\right|^{2}\right]
$$

Selecting $k_{i}(x, r, e)$ as given by Eq. (35) to cancel the indefinite terms, ensures $\dot{V}_{e} \leq-\sum_{i=1}^{n} \frac{\lambda_{i}}{2} r_{i}^{2} e_{i}^{2}$, which proves that $r_{i}(t) \in \mathcal{L}_{\infty}$ and $\lim _{t \rightarrow \infty} e_{i}(t)=0$. The functions $k_{i}(x, r, e)$ contain a nonlinear damping term to achieve boundedness of $r_{i}$, but the constant $\epsilon$ multiplying the damping term can be chosen arbitrarily small.

This completes the design of the estimator, which consists of output filters Eq. (27), update laws Eq. (28) and dynamic scalings Eq. (34). Note that the estimator, in general, employs overparametrization, which is not necessarily disadvantageous from a performance point of view. However, in a numerical implementation it can lead to a higher computational load. The total order of the estimator is $\sum_{i=1}^{n} p_{i}+2 n$.

\section{III.B. Control Law Design}

The properties of the estimator can be exploited by designing an adaptive control law. In this study a backstepping controller is used to stabilize the closed-loop system. The command filtered backstepping design procedure ${ }^{5,12}$ starts by defining the tracking errors as

$$
z_{i}=x_{i}-x_{i, r}, \quad i=1, \ldots, n
$$

where $x_{i, r}$ are the intermediate control laws to be designed. The compensated tracking errors are defined as

$$
\bar{z}_{i}=z_{i}-\chi_{i}
$$

with the signals $\chi_{i}$ to be defined. The dynamics of $\bar{z}_{i}$ can be written as

$$
\begin{aligned}
\dot{\bar{z}}_{i} & =z_{i+1}+x_{i+1, r}+\varphi_{i}^{T} \theta_{i}-\dot{\chi}_{i} \\
\dot{\bar{z}}_{n} & =u+\varphi_{n}^{T} \theta_{n}-\dot{x}_{n, r}-\dot{\chi}_{n} .
\end{aligned}
$$


The idea is now to design a control law that renders the closed-loop system $\mathcal{L}_{2}$ stable from the 'perturbation' inputs $\varphi_{i}^{T} \sigma_{i}$ to the output $\bar{z}_{1}$ and keeps all signals bounded. To stabilize Eq. (38) the following desired (intermediate) controls are proposed:

$$
\begin{aligned}
x_{i+1, r}^{0} & =-\kappa_{i}+\bar{z}_{i-1}-\varphi_{i}^{T}\left(\hat{\theta}_{i}+\beta_{i}\right)-\chi_{i+1}, \quad i=1, \ldots, n-1, \\
u^{0} & =-\kappa_{n}+\bar{z}_{n-1}-\varphi_{n}^{T}\left(\hat{\theta}_{n}+\beta_{n}\right)+\dot{x}_{n, r}
\end{aligned}
$$

with the stabilizing functions $\kappa_{i}$ given as

$$
\kappa_{i}=\bar{c}_{i} z_{i}+\frac{\mu r_{i}^{2}}{2} \bar{z}_{i}+\bar{k}_{i} \bar{\lambda}_{i}
$$

for $i=1, \ldots, n$, where $\bar{c}_{i}>0, \mu>0$ and $\bar{k}_{i} \geq 0$ are constants. The integral terms are defined as

$$
\bar{\lambda}_{i}=\int_{0}^{t} \bar{z}_{i}(t) d t
$$

The desired (intermediate) control laws Eq. (39) are fed through second order low pass filters to produce the actual intermediate controls $x_{i+1, r}, u$ and their derivatives. ${ }^{25}$ The use of command filters avoids the tedious analytical calculations of the derivatives of the intermediate control in subsequent design steps. An additional advantage is that the filters can be used to enforce magnitude or rate limits on the intermediate states and control. ${ }^{4}$ The effect that the use of these filters has on the tracking errors can be captured with the stable linear filters

$$
\begin{aligned}
\dot{\chi}_{i} & =-\bar{c}_{i} \chi_{i}+\left(x_{i+1, r}-x_{i+1, r}^{0}\right), \quad i=1, \ldots, n-1 \\
\dot{\chi}_{n} & =-\bar{c}_{n} \chi_{n}+\left(u-u^{0}\right) .
\end{aligned}
$$

The stability properties of the adaptive control framework based on this command filtered backstepping controller in combination with the I\&I based estimator design of Lemma III.1 can be proved using the control Lyapunov function

$$
V_{c}(\bar{z}, \sigma)=\sum_{i=1}^{n}\left(\bar{z}_{i}^{2}+\frac{1}{2} \bar{k} \bar{\lambda}_{i}^{2}+\sigma_{i}^{T} \sigma_{i}\right) .
$$

Taking the time derivative of $V_{c}$ and following some of the steps used in the proof of Lemma III.1 results in

$$
\begin{aligned}
\dot{V}_{c} \leq & 2 \sum_{i=1}^{n-1} \bar{z}_{i}\left(z_{i+1}+x_{i+1, r}+\varphi_{i}^{T} \theta_{i}-\dot{\chi}_{i}\right)+2 \bar{z}_{n}\left(u+\varphi_{n}^{T} \theta_{n}-\dot{x}_{n, r}-\dot{\chi}_{n}\right) \\
& +\sum_{i=1}^{n} \bar{k} \bar{\lambda}_{i} \bar{z}_{i}-\sum_{i=1}^{n}\left(2 \gamma_{i}-\frac{1}{2}\right)\left(\varphi_{i}^{T} \sigma_{i}\right)^{2} \\
= & -2 \sum_{i=1}^{n} \bar{z}_{i}\left(\bar{c}_{i} \bar{z}_{i}+\frac{\mu_{i} r_{i}^{2}}{2} \bar{z}_{i}+\varphi_{i}^{T} \sigma_{i} r_{i}\right)-\sum_{i=1}^{n}\left(2 \gamma_{i}-\frac{1}{2}\right)\left(\varphi_{i}^{T} \sigma_{i}\right)^{2} \\
= & -2 \sum_{i=1}^{n} \bar{c}_{i} \bar{z}_{i}^{2}-\sum_{i=1}^{n}\left[\frac{1}{\sqrt{\mu}} \varphi_{i}^{T} \sigma_{i}+\sqrt{\mu} r_{i} \bar{z}_{i}\right]^{2}-\sum_{i=1}^{n}\left(2 \gamma_{i}-\frac{1}{2}-\frac{1}{\mu}\right)\left(\varphi_{i}^{T} \sigma_{i}\right)^{2} \\
\leq & -2 \sum_{i=1}^{n} \bar{c}_{i} \bar{z}_{i}^{2}-\sum_{i=1}^{n}\left(2 \gamma_{i}-\frac{1}{2}-\frac{1}{\mu}\right)\left(\varphi_{i}^{T} \sigma_{i}\right)^{2}
\end{aligned}
$$

It can be concluded that, if $\gamma_{i} \geq \frac{\mu+2}{4 \mu}$, the closed-loop system consisting of Eqs. (25), (39) and the I\&I based estimator of the previous section, which consists of output filters Eq. (27), update laws Eq. (28) and dynamic scalings Eq. (34), has a globally stable equilibrium. Furthermore, by Barbalat's lemma $\lim _{t \rightarrow \infty} \bar{z}_{i}=0$ and $\lim _{t \rightarrow \infty} \varphi_{i}^{T} \sigma_{i}=0$. When the command filters are properly designed, i.e. with bandwidths sufficiently high, and no rate or magnitude limits are in effect, $\bar{z}_{i}$ will converge to the close neighborhood of the real tracking errors $z_{i}$. This concludes the discussion on the modular I\&I based adaptive backstepping control design. The method will now be demonstrated and validated in a nonlinear flight control design example. 


\section{Aircraft Model Description}

The aircraft dynamic model used for this study was obtained from Ref. 26 and resembles a simplified F-18 model. The model has seven independent control surfaces, i.e. left and right elevators, left and right ailerons, leading and trailing edge flaps, and collective rudders. All control inputs are defined positive in the conventional way, e.g. a positive rudder deflection $\delta_{r}$ decreases the yaw rate $r$. A layout of the aircraft and its control surfaces can be seen in Fig. 2. The main simplifications made in the dynamic model are constant airspeed and no lift or drag effects on the control surfaces. The aircraft model includes second order actuator dynamics. The magnitude, rate and bandwidth limits of the actuators are specified in Table 1 . The relevant equations of motion are given by

Table 1. Actuator and Effector Constraints

\begin{tabular}{cccc}
\hline \hline Effector & $\begin{array}{c}\text { Position Limit } \\
{[\mathrm{deg}]}\end{array}$ & $\begin{array}{c}\text { Rate Limit } \\
{[\mathrm{deg} / \mathrm{s}]}\end{array}$ & $\begin{array}{c}\text { Bandwidth } \\
{[\mathrm{rad} / \mathrm{s}]}\end{array}$ \\
\hline Elevator & {$[-24,10.5]$} & \pm 40 & 50 \\
Ailerons & {$[-25,45]$} & \pm 100 & 50 \\
Leading Edge Flaps & {$[-3,33]$} & \pm 15 & 50 \\
Trailing Edge Flaps & {$[-8,45]$} & \pm 18 & 50 \\
Rudder & {$[-30,30]$} & \pm 82 & 50 \\
\hline \hline
\end{tabular}

$$
\begin{aligned}
\frac{d}{d t}\left(\begin{array}{c}
\phi \\
\alpha \\
\beta \\
\theta \\
p \\
q \\
r
\end{array}\right)= & \left.\begin{array}{c}
p+q \tan \theta \sin \phi+r \tan \theta \cos \phi \\
q-p \beta+z_{\alpha} \Delta \alpha+\left(g_{0} / V\right)\left(\cos \theta \cos \phi-\cos \theta_{0}\right) \\
y_{\beta} \beta+p\left(\sin \alpha_{0}+\Delta \alpha\right)-r \cos \alpha_{0}+\left(g_{0} / V\right) \cos \theta \sin \phi \\
q \cos \phi-r \sin \theta \\
l_{\beta} \beta+l_{q} q+l_{r} r+\left(l_{\beta \alpha} \beta+l_{r \alpha} r\right) \Delta \alpha+l_{p} p-i_{1} q r \\
m_{\alpha} \Delta \alpha+m_{q} q+i_{2} p r-m_{\dot{\alpha}} p+m_{\dot{\alpha}}\left(g_{0} / V\right)\left(\cos \theta \cos \phi-\cos \theta_{0}\right)
\end{array}\right) \\
& +\left(\begin{array}{ccccccc}
0 & 0 & 0 & 0 & 0 & 0 & 0 \\
0 & 0 & 0 & 0 & 0 & 0 & 0 \\
0 & 0 & 0 & 0 & 0 & 0 & 0 \\
0 & 0 & 0 & 0 & 0 & 0 & 0 \\
l_{\delta_{e l}} & l_{\delta_{e r}} & l_{\delta_{a l}} & l_{\delta_{a r}} & 0 & 0 & l_{\delta_{r}} \\
m_{\delta_{e l}} & m_{\delta_{e r}} & m_{\delta_{a l}} & m_{\delta_{a r}} & m_{\delta_{l e f}} & m_{\delta_{\text {tef }}} & m_{\delta_{r}} \\
n_{\delta_{e l}} & n_{\delta_{e r}} & n_{\delta_{a l}} & n_{\delta_{a r}} & 0 & 0 & n_{\delta_{r}}
\end{array}\right)\left(\begin{array}{c}
\delta_{e l} \\
\delta_{e r} \\
\delta_{a l} \\
\delta_{a r} \\
\delta_{l e f} \\
\delta_{\text {tef }} \\
\delta_{r}
\end{array}\right)
\end{aligned}
$$

Aerodynamic data for the model is available in Tables 2 and 3 of the Appendix for two trimmed flight conditions: flight condition 1 at an altitude of $30000 \mathrm{ft}$ and a Mach number of 0.7 , and flight condition 2 at $40000 \mathrm{ft}$ altitude and a Mach number of 0.6.

\section{Adaptive Flight Control Design}

In this section the theory discussed in Section III is used to construct a nonlinear adaptive flight control law for the aircraft model Eq. (42). The control objective is to track smooth reference signals with $\phi, \alpha$ and $\beta$. It is assumed that all stability and control derivatives are unknown. A scheme of the proposed adaptive flight controller is depicted in Figure 3.

Before, the adaptive control design procedure begins, the aircraft dynamic model Eq. (42) is rewritten in a more general form. Define the states $x_{1}=\phi, x_{2}=\alpha, x_{3}=\beta, x_{4}=\theta, x_{5}=p, x_{6}=q, x_{7}=r$ and the control inputs $u=\left(\delta_{e l}, \delta_{e r}, \delta_{a l}, \delta_{a r}, \delta_{l e f}, \delta_{t e f}, \delta_{r}\right)^{T}$, then the system Eq. (42) can be rewritten as

$$
\dot{x}_{i}=f_{i}(x)+\varphi_{i}(x, u)^{T} \theta_{i}, \quad i=1, \ldots, 7,
$$




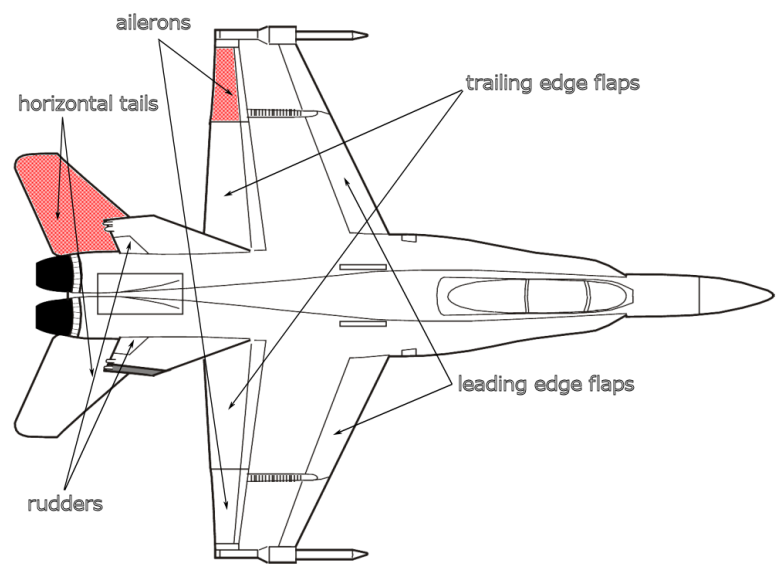

Figure 2. The control surfaces of the aircraft model. The control surfaces which will lock in place during the simulation scenarios are indicated.

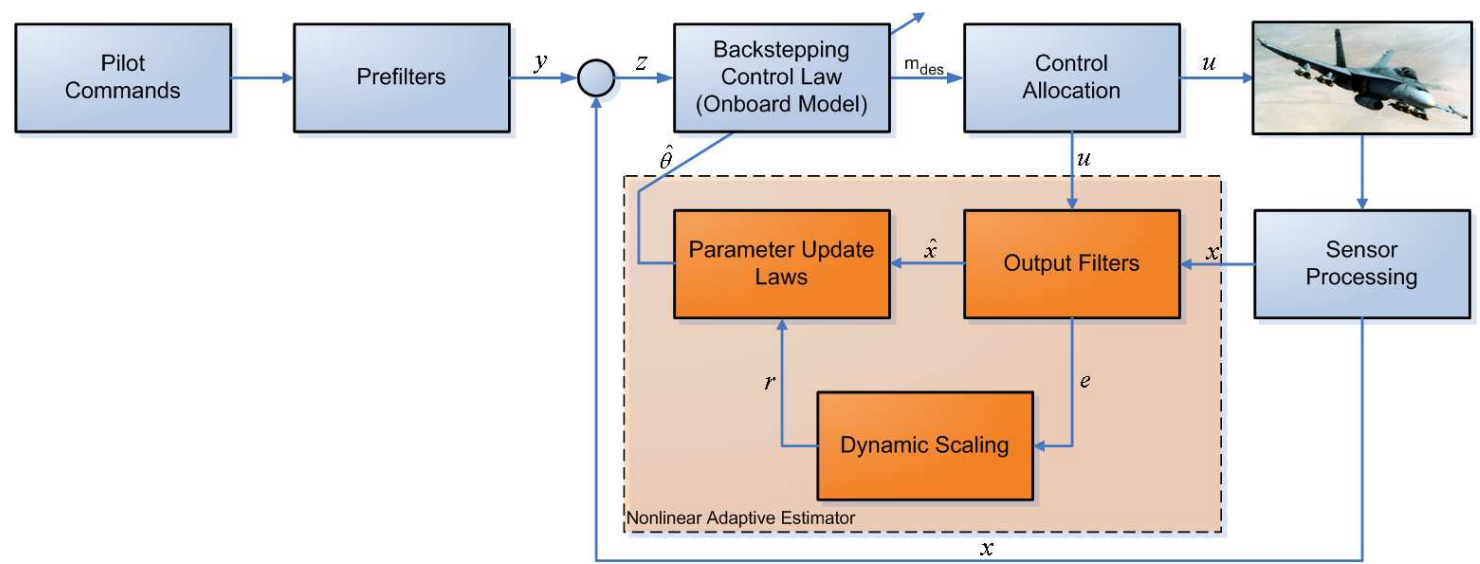

Figure 3. Nonlinear adaptive control framework.

with the known functions

$$
\begin{aligned}
f_{1}(x) & =x_{5}+x_{6} \tan x_{4} \sin x_{1}+x_{7} \tan x_{4} \cos x_{1}, \\
f_{2}(x) & =x_{6}-x_{5} x_{3}+\frac{g_{0}}{V}\left(\cos x_{4} \cos x_{1}-\cos \theta_{0}\right), \\
f_{3}(x) & =x_{5}\left(\sin \alpha_{0}+\left(x_{2}-\alpha_{0}\right)\right)-x_{7} \cos \alpha_{0}+\frac{g_{0}}{V} \cos x_{4} \sin x_{1}, \\
f_{4}(x) & =x_{6} \cos x_{1}-x_{7} \sin x_{4}, \quad f_{5}(x)=-i_{1} x_{6} x_{7}, \quad f_{6}(x)=i_{2} x_{5} x_{7}, \quad f_{7}(x)=-i_{3} x_{5} x_{6},
\end{aligned}
$$

the unknown parameter vectors

$$
\begin{aligned}
& \theta_{1}=0, \quad \theta_{2}=z_{\alpha}, \quad \theta_{3}=y_{\beta}, \quad \theta_{4}=0 \\
& \theta_{5}=\left(l_{p}, l_{q}, l_{r}, l_{\beta \alpha}, l_{r \alpha}, l_{0}, l_{\delta_{e l}}, l_{\delta_{e r}}, l_{\delta_{a l}}, l_{\delta_{a r}}, l_{\delta_{r}}\right)^{T}, \\
& \theta_{6}=\left(m_{\alpha}, m_{q}, m_{\dot{\alpha}}, m_{0}, m_{\delta_{e l}}, m_{\delta_{e r}}, m_{\delta_{a l}}, m_{\delta_{a r}}, m_{\delta_{l e f}}, m_{\delta_{t e f}}, m_{\delta_{r}}\right)^{T}, \\
& \theta_{7}=\left(n_{\beta}, n_{p}, n_{q}, n_{r}, n_{p \alpha}, n_{0}, n_{\delta_{e l}}, n_{\delta_{e r}}, n_{\delta_{a l}}, n_{\delta_{a r}}, n_{\delta_{r}}\right)^{T},
\end{aligned}
$$

and the regressors

$$
\begin{aligned}
& \varphi_{1}(x, u)=0, \quad \varphi_{2}(x, u)=x_{2}-\alpha_{0}, \quad \varphi_{3}(x, u)=x_{3}, \quad \varphi_{4}(x, u)=0, \\
& \varphi_{5}(x, u)=\left(x_{5}, x_{6}, x_{7}, x_{3}\left(x_{2}-\alpha_{0}\right), x_{7}\left(x_{2}-\alpha_{0}\right), 1, u_{1}, u_{2}, u_{3}, u_{4}, u_{7}\right)^{T}, \\
& \varphi_{6}(x, u)=\left(x_{2}-\alpha_{0}, x_{6}, x_{5} x_{3}+\frac{g_{0}}{V}\left(\cos x_{4} \cos x_{1}-\cos \theta_{0}\right), 1, u_{1}, \ldots, u_{7}\right)^{T}, \\
& \varphi_{7}(x, u)=\left(x_{3}, x_{5}, x_{6}, x_{7}, x_{5}\left(x_{2}-\alpha_{0}\right), 1, u_{1}, u_{2}, u_{3}, u_{4}, u_{7}\right)^{T} .
\end{aligned}
$$

\section{1 of 18}


Note that the parameters $l_{0}, m_{0}$ and $n_{0}$ have been added to the unknown parameter vectors to compensate for any additional moments caused by failures, e.g. actuator hard-overs.

\section{V.A. Estimator Design}

The I\&I estimator design of Section III.A can be applied directly to the rewritten aircraft equations of motion Eq. (43). Following the estimator design procedure of Section III.A, the scaled estimation errors are defined as

$$
\sigma_{i}=\frac{\hat{\theta}_{i}-\theta_{i}+\beta_{i}\left(x_{i}, \hat{x}\right)}{r_{i}}, \quad i=2,3,5,6,7 .
$$

Let the output errors be given by $e_{i}=\hat{x}_{i}-x_{i}$, then the output filters are defined as

$$
\dot{\hat{x}}_{i}=f_{i}+\varphi_{i}^{T}\left(\hat{\theta}_{i}+\beta_{i}\right)-k_{i} e_{i}, \quad i=2,3,5,6,7 .
$$

Note that no output filters are needed for $x_{1}$ - and $x_{4}$-dynamics, since they contain no uncertainties. The estimator dynamics are given by

$$
\dot{\hat{\theta}}_{i}=-\frac{\partial \beta_{i}}{\partial x_{i}}\left(\hat{x}_{i}+k_{i} e_{i}\right)-\sum_{j=1}^{7} \frac{\partial \beta_{i}}{\partial \hat{x}_{j}} \dot{\hat{x}}_{j}-\sum_{k=1}^{7} \frac{\partial \beta_{i}}{\partial u_{k}} \dot{u}_{k},
$$

where the functions $\beta_{i}\left(x_{i}, \hat{x}\right)$ are obtained from Eq. (30), i.e.

$$
\begin{aligned}
& \beta_{2}=\gamma_{2}\left(\frac{1}{2} x_{2}^{2}-\alpha_{0} x_{2}\right), \quad \beta_{3}=\gamma_{3} \frac{1}{2} x_{3}^{2}, \\
& \beta_{5}=\gamma_{5} x_{5}\left(\hat{x}_{3}, \hat{x}_{6}, \hat{x}_{7}, \hat{x}_{3}\left(\hat{x}_{2}-\alpha_{0}\right), \hat{x}_{7}\left(\hat{x}_{2}-\alpha_{0}\right), \frac{1}{2} x_{5}, 1, u_{1}, u_{2}, u_{3}, u_{4}, u_{7}\right)^{T}, \\
& \beta_{6}=\gamma_{6} x_{6}\left(\hat{x}_{2}-\alpha_{0}, \frac{1}{2} x_{6},-\hat{x}_{5} \hat{x}_{3}+\frac{g_{0}}{V}\left(\cos x_{4} \cos x_{1}-\cos \theta_{0}\right), 1, u_{1}, \ldots, u_{7}\right)^{T}, \\
& \beta_{7}=\gamma_{7} x_{7}\left(\hat{x}_{3}, \frac{1}{2} x_{7}, \hat{x}_{5}, \hat{x}_{5}\left(\hat{x}_{2}-\alpha_{0}\right), \hat{x}_{6}, 1, u_{1}, u_{2}, u_{3}, u_{4}, u_{7}\right)^{T},
\end{aligned}
$$

with $\gamma_{i}>0$. Taking the derivative of these functions results in

$$
\begin{aligned}
\dot{\beta}_{2} & =\gamma_{2} \varphi_{2}, \quad \dot{\beta}_{3}=\gamma_{3} \varphi_{3}, \\
\dot{\beta}_{5} & =\gamma_{5} \varphi_{3}-\gamma_{5} e_{2}\left(0,0,0,0,-x_{3},-x_{7}, 0,0,0,0,0,0\right)^{T}-\gamma_{5} e_{3}\left(0,-1,0,0, \alpha_{0}-x_{2}-e_{2}, 0,0,0,0,0,0,0\right)^{T} \\
& -\gamma_{5} e_{6}(0,0,-1,0,0,0,0,0,0,0,0,0)^{T}-\gamma_{5} e_{7}\left(0,0,0,-1,0, \alpha_{0}-x_{2}-e_{2}, 0,0,0,0,0,0\right)^{T}+\sum_{k=1} \frac{\partial \beta_{5}}{\partial u_{k}} \dot{u}_{k} \\
& =\gamma_{5} \varphi_{5}-\gamma_{5} e_{2} \delta_{52}-\gamma_{5} e_{3} \delta_{53}-\gamma_{5} e_{6} \delta_{56}-\gamma_{5} e_{7} \delta_{57}+\sum_{k=1}^{7} \frac{\partial \beta_{5}}{\partial u_{k}} \dot{u}_{k}, \\
\dot{\beta}_{6} & =\gamma_{6} \varphi_{6}-\gamma_{6} e_{2}(0,-1,0,0,0,0,0,0,0,0,0)^{T}-\gamma_{6} e_{3}\left(0,0,0, x_{5}+e_{5}, 0,0,0,0,0,0,0\right)^{T} \\
& -\gamma_{6} e_{5}\left(0,0,0, x_{3}, 0,0,0,0,0,0,0\right)^{T}+\sum_{k=1}^{7} \frac{\partial \beta_{6}}{\partial u_{k}} \dot{u}_{k} \\
& =\gamma_{6} \varphi_{6}-\gamma_{6} e_{2} \delta_{62}-\gamma_{6} e_{3} \delta_{63}-\gamma_{6} e_{5} \delta_{65}+\sum_{k=1}^{7} \frac{\partial \beta_{6}}{\partial u_{k}} \dot{u}_{k}, \\
\dot{\beta}_{7} & =\gamma_{7} \varphi_{7}-\gamma_{7} e_{2}\left(0,0,0,0,-x_{5}, 0,0,0,0,0,0\right)^{T}-\gamma_{7} e_{3}(0,-1,0,0,0,0,0,0,0,0,0)^{T} \\
& -\gamma_{7} e_{5}\left(0,0,0,-1, \alpha_{0}-x_{2}-e_{2}, 0,0,0,0,0,0\right)^{T}-\gamma_{7} e_{6}(0,0,0,0,0,-1,0,0,0,0,0)^{T}+\sum_{k=1}^{7} \frac{\partial \beta_{7}}{\partial u_{k}} \dot{u}_{k} \\
& =\gamma_{7} \varphi_{7}-\gamma_{7} e_{2} \delta_{72}-\gamma_{7} e_{3} \delta_{73}-\gamma_{7} e_{5} \delta_{75}-\gamma_{7} e_{6} \delta_{76}+\sum_{k=1}^{7} \frac{\partial \beta_{7}}{\partial u_{k}} \dot{u}_{k},
\end{aligned}
$$


where the bracketed terms correspond to the functions $\delta_{i j}(x, e)$ given by Eq. (31). Finally, from Eqs. (34) and (35) the dynamic scaling parameters $r_{i}$ and the gains $k_{i}$ are given by

$$
\dot{r}_{i}=\frac{\gamma_{i}}{2} r_{i} \sum_{j=1}^{7} e_{j}^{2}\left|\delta_{i j}(x, e)\right|^{2}
$$

and

$$
k_{i}(x, r, e)=\lambda_{i} r_{i}^{2}+\epsilon \sum_{j=1}^{7} c_{j} r_{j}^{2}\left|\delta_{j i}(x, e)\right|^{2},
$$

with $\lambda_{i}>0, \epsilon>0$ and $r_{i}(0)=1$. This completes the nonlinear estimator design for the aircraft model.

\section{V.B. Backstepping Control Design}

An inner-outer loop backstepping controller is now constructed to exploit the properties of the estimator. Since the backstepping flight controller is designed for a multivariable system, it is more convenient to rewrite the system Eq. (42) in a vector form as

$$
\begin{aligned}
& \dot{X}_{1}=F_{1}\left(X_{1}, X_{u}\right)+\Phi_{1}\left(X_{1}, X_{u}\right)^{T} \Theta+B_{1}\left(X_{1}, X_{u}\right) X_{2} \\
& \dot{X}_{2}=F_{2}\left(X_{1}, X_{2}, X_{u}\right)+\Phi_{21}\left(X_{1}, X_{2}, X_{u}\right)^{T} \Theta+\Phi_{22}\left(X_{1}, X_{2}, X_{u}\right)^{T} \Theta U \\
& \dot{X}_{u}=F_{u}\left(X_{1}, X_{2}, X_{u}\right)
\end{aligned}
$$

where $X_{1}=(\phi, \alpha, \beta)^{T}, X_{2}=(p, q, r)^{T}, U=\left(\delta_{e l}, \delta_{e r}, \delta_{a l}, \delta_{a r}, \delta_{l e f}, \delta_{t e f}, \delta_{r}\right)^{T}$, and the uncontrolled state $X_{u}=\theta$. The known nonlinear aircraft dynamics are represented by the vector functions $F_{1}(X), F_{2}(X)$ and $F_{u}(X)$ and the matrix function $B_{1}(X)$. The functions $\Phi_{1}(X), \Phi_{21}(X)$ and $\Phi_{22}(X)$ are the regressor matrices, while $\Theta$ is a vector containing the unknown stability and control derivatives of the system, defined as

$$
\Theta=\left(\theta_{2}^{T}, \theta_{3}^{T}, \theta_{5}^{T}, \theta_{6}^{T}, \theta_{7}^{T}\right)^{T}
$$

Now that the system has been rewritten in a structured form the actual control design procedure can be discussed. The control objective is to track a smooth reference signal $Y_{r}$ with state vector $X_{1}$. The reference $X_{1, r}$ and its derivative $\dot{X}_{1, r}$ are generated by a linear second order filter. The static feedback control design can be divided in two parts, an outer loop to control the aerodynamic angles using the angular rates, and an inner loop to control the angular rates using the control surfaces. Following the design procedure of Section III.B the tracking errors are defined as

$$
Z_{i}=X_{i}-X_{i, r}, \quad i=1,2
$$

and the modified tracking errors as

$$
\bar{Z}_{i}=Z_{i}-\Xi_{i}
$$

To stabilize the outer loop the desired intermediate control is defined as

$$
X_{2, r}^{0}=B_{1}^{-1}\left(-F_{1}-\Phi_{1}^{T}(\hat{\Theta}+\beta(X))-C_{1} Z_{1}-K_{1} \Lambda_{1}\right),
$$

with $C_{1}=C_{1}^{T}>0, K_{1}=K_{1}^{T} \geq 0$, where $\hat{\Theta}$ is the estimate of $\Theta$ obtained from the I\&I based estimator and

$$
\beta(X)=\left(\beta_{2}^{T}, \beta_{3}^{T}, \beta_{5}^{T}, \beta_{6}^{T}, \beta_{7}^{T}\right)^{T} .
$$

The integral term $\Lambda_{1}$ is given as

$$
\Lambda_{1}=\int_{0}^{t} \bar{Z}_{1}(t) d t .
$$

The desired intermediate control law Eq. (39) is now fed through second order low pass filters to produce the actual intermediate control $X_{2, r}$ and its derivatives. The effect that the use of these filters has on the tracking errors can be captured with the stable linear filter

$$
\dot{\Xi}_{1}=-C_{1} \Xi_{1}+B_{1}\left(X_{2, r}-X_{2, r}^{0}\right) \text {. }
$$


The design of the inner loop starts at the $Z_{2}$-subsystem given by

$$
\dot{Z}_{2}=F_{2}+\Phi_{2}^{T} \Theta+B_{2} U-\dot{X}_{2, r} .
$$

To stabilize the system Eq. (50) the desired control $U^{0}$ is defined as

$$
U^{0}=\left(\Phi_{21}^{T}(\hat{\Theta}+\beta(X))\right)^{\dagger}\left(-B_{1}^{T} \bar{Z}_{1}-C_{2} Z_{2}-F_{2}-\Phi_{21}^{T}(\hat{\Theta}+\beta(X))+\dot{X}_{2, r}\right),
$$

with $C_{2}=C_{2}^{T}>0$, and where $\left(\Phi_{21}^{T}(\hat{\Theta}+\beta(X))\right)^{\dagger}$ represents the pseudo-inverse of the estimate of the control effectiveness matrix. Alternatively, a more sophisticated control allocation method could be used, see e.g. Ref. 27. The parameter projection method is used to avoid singularity problems. The actual control $U$ is found by filtering $U_{0}$. Finally, the stable linear filter

$$
\dot{\Xi}_{2}=-C_{2} \Xi_{2}+\hat{B}_{2}\left(U-U^{0}\right)
$$

is defined. This concludes the design of the adaptive flight controller for the over-actuated fighter aircraft model Eq. (42). The tracking performance and parameter estimation capabilities of the controller can now be evaluated in numerical simulations.

\section{Numerical Simulations and Results}

This section presents the simulation results from the application of the adaptive flight controller developed in the previous section to the over-actuated fighter aircraft model of Section IV implemented in the MATLAB/Simulink ${ }^{\circledR}$ environment. The simulations are performed at the two mentioned flight conditions for which the aerodynamic data can be found in Tables 2 and 3. Sensor models obtained from Ref. 28 and transport delays of $20 \mathrm{~ms}$ have been added to the controller to model an onboard computer implementation of the control laws.

The backstepping controller is tuned in a trial-and-error procedure on the nominal aircraft model. The final gains were chosen as $C_{1}=2 I, C_{2}=5 I, K_{1}=0.1 I$, where $I$ represents the $3 \times 3$ identity matrix.

Tuning of the estimator is relatively straight-forward, since increasing the adaptation gains $\gamma_{i}$ not only increases the adaptation rate but also improves the closed-loop performance. This is in contrast with the integrated adaptive backstepping approaches ${ }^{5,11}$ where increasing the adaptation gains can lead to a worsened transient performance. The influence of the size of the other estimator parameters, $\lambda_{i}$ and $\epsilon$, on the performance is very limited, simply selecting them larger than zero is enough to guarantee convergence of the filtered states to the true states and boundedness of the dynamic scaling parameters. The final gain and parameter selection is: $\gamma_{i}=10, \lambda_{i}=0.01, i=1, \ldots, 7$, and $\epsilon=0.01$.

\section{VI.A. Simulation with Left Aileron Runaway}

In this first simulation a mixed maneuver involving a series of angle of attack and roll angle doublets is considered. The aircraft model starts at flight condition 2 in a trimmed horizontal flight where after 1 second of simulation time the left aileron suffers a hard-over failure and moves to its limit of 45 degrees. This failure results in a large additional rolling moment and minor additional pitch and yawing moments. Note that the adaptive controller does not use any sensor measurements of the control surface position or any other form of fault detection. The results of this simulation can be found in Figure 4.

The adaptive controller manages to rapidly return the states to their reference values after the failure. As can be expected, the coupling between longitudinal and lateral motion is more prominent in the response after the failure. It can be seen in Figure 4(c) that the total moment coefficients post-failure are estimated rapidly and accurately. However, the individual parameters have not converged to their true values since this maneuver alone does not provide the estimator with enough information.

In Figure 4(d) some additional parameters of the I\&I estimator are plotted, i.e. the dynamic scaling parameters $r_{*}$, the output filter states $\hat{x}$ and the prediction errors $e_{*}$. All signals are behaving as they should be, the dynamic scalings converge to constant values, the filter states follow the aircraft model states and the prediction errors converge to zero. In the control surface plots it can be seen that most of the additional moment is compensated by the right aileron and the left elevator. The simple pseudo-inverse control allocation scheme does not give any preference to certain control surfaces or axes. The tracking performance and parameter convergence of the adaptive controller are very good for this failure case. 


\section{VI.B. Simulation with Left Elevator Runaway}

The second simulation is again of a mixed maneuver involving a series of angle of attack and roll angle doublets at flight condition 2. The aircraft model starts in a straight, horizontal flight where after 1 second of simulation time the left elevator suffers a hard-over failure and moves to its limit of 10.5 degrees. The simulation results of this maneuver can be found in Figure 5, which is again divided in 4 subplots.

The results demonstrate again that the adaptive controller performs excellent. The total moment coefficients are rapidly found by the estimator and tracking performance is excellent. However, the individual components of the parameter estimate vectors again do not converge to their true values.

\section{Conclusions}

In this paper, a nonlinear adaptive flight control design framework based on the concept of immersion and invariance (I\&I) in combination with adaptive backstepping is discussed. Global asymptotic stability of the resulting closed-loop system and convergence of the parameter estimation errors is proved using Lyapunov theory. The main advantage of a controller synthesized with the I\&I based modular adaptive backstepping approach over a conventional adaptive backstepping controller is that it is much easier to tune, since it allows for prescribed stable dynamics to be assigned to the parameter estimation error. Furthermore, this approach does not suffer from undesired transient performance resulting from unexpected dynamical behavior of the parameter update laws. Moreover, the I\&I based modular adaptive backstepping approach has even stronger provable stability and convergence properties than the conventional, integrated adaptive backstepping approaches, while at the same time achieving a modularity in the design of the controller and identifier modules. As an example a modular adaptive backstepping flight controller with I\&I based estimator is designed for a simplified nonlinear fighter aircraft model. Numerical simulations were performed to verify the performance of the adaptive control law. Actuator hard-over failures were introduced to evaluate the control performance and the parameter estimation process. The results show that the adaptive flight controller manages good tracking performance while in the presence of actuator saturation, despite these serious failures.

Future research will focus on the application of the method to more complex and realistic flight control problems.

\section{References}

${ }^{1}$ Steinberg, M. L. and Page, A. B., "Nonlinear Adaptive Flight Control with a Backstepping Design Approach," AIAA98-4230, 1998.

${ }^{2}$ Calise, A. J., Lee, S., and Sharma, M., "Development of a Reconfigurable Flight Control Law for Tailless Aircraft," Journal of Guidance, Control and Dynamics, Vol. 24, No. 5, Sep.-Oct. 2001, pp. 896-902.

${ }^{3}$ Shin, D. H. and Kim, Y., "Reconfigurable Flight Control System Design Using Adaptive Neural Networks," IEEE Transactions on Control Systems Technology, Vol. 12, No. 1, Jan. 2004, pp. 87-100.

${ }^{4}$ Farrell, J., Sharma, M., and Polycarpou, M., "Backstepping Based Flight Control with Adaptive Function Approximation," AIAA Journal of Guidance, Control and Dynamics, Vol. 28, No. 6, Jan. 2005, pp. 1089-1102.

${ }^{5}$ Sonneveldt, L., Chu, Q. P., and Mulder, J. A., "Nonlinear Flight Control Design Using Constrained Adaptive Backstepping," Journal of Guidance, Control, and Dynamics, Vol. 30, No. 2, Mar-Apr 2007, pp. 322-336.

${ }^{6}$ Lavretsky, E., Hovakimyan, N., and Cao, C., "Adaptive Design for Uncertain Systems with Nonlinear-in-Control Dynamics," AIAA Guidance, Navigation, and Control Conference and Exhibit, 2007.

${ }^{7}$ Wang, J., Patel, V., Cao, C., Hovakimyan, N., and Lavretsky, E., "Novel L1 Adaptive Control Methodology for Aerial Refueling with Guaranteed Transient Performance," Journal of Guidance, Control and Dynamics, Vol. 31, 2008, pp. $182-193$.

${ }^{8}$ Lombaerts, T., Huisman, H., Chu, Q., Mulder, J. A., and Joosten, D., "Nonlinear Reconfiguring Flight Control Based on Online Physical Model Identification," Journal of Guidance, Control, and Dynamics, Vol. 32, 2009, pp. $727-748$.

${ }^{9}$ Nguyen, N. and Krishnakumar, K., "Hybrid Intelligent Flight Control with Adaptive Learning Parameter Estimation," Journal of Aerospace Computing, Information, and Communication, Vol. 6, 2009, pp. 171-186.

${ }^{10}$ Kannelakopoulos, I., Kokotović, P. V., and Morse, A. S., "Systematic Design of Adaptive Controllers for Feedback Linearizable Systems," IEEE Transactions on Automatic Control, Vol. 36, No. 11, Nov. 1991, pp. 1241-1253.

${ }^{11}$ Krstić, M., Kanellakopoulos, I., and Kokotović, P. V., "Adaptive Nonlinear Control Without Overparametrization," Systems and Control Letters, Vol. 19, sept. 1992, pp. 177-185.

${ }^{12}$ Sonneveldt, L., van Oort, E., Chu, Q., de Visser, C., Mulder, J., and Breeman, J., "Lyapunov-based Fault Tolerant Flight Control Designs for a Modern Fighter Aircraft Model," AIAA Guidance, Navigation, and Control Conference and Exhibit, 2009.

${ }^{13}$ Krstić, M. and Kokotović, P. V., "Adaptive Nonlinear Design with Controller-Identifier Separation and Swapping," IEEE Transactions on Automatic Control, Vol. 40, No. 3, March 1995, pp. 426-440. 
${ }^{14}$ van Oort, E. R., Sonneveldt, L., Chu, Q. P., and Mulder, J. A., "A Comparison of Adaptive Nonlinear Control Designs for an Over-Actuated Fighter Aircraft Model," AIAA Guidance, Navigation and Control Conference and Exhibit, 2008.

${ }^{15}$ Karagiannis, D. and Astolfi, A., "Nonlinear Observer Design Using Invariant Manifolds and Applications," 44 th IEEE Conf. Decision and Control, 2005.

${ }^{16}$ Karagiannis, D. and Astolfi, A., "Nonlinear Adaptive Control of Systems in Feedback Form: An Alternative to Adaptive Backstepping," Systems and Control Letters, Vol. 57, 2008, pp. 733-739.

${ }^{17}$ Carnevale, D., Karagiannis, D., and Astolfi, A., "Reduced-order Observer Design for Nonlinear Systems," European Control Conference, 2007.

${ }^{18}$ Karagiannis, D. and Astolfi, A., "Observer Design for a Class of Nonlinear Systems using Dynamic Scaling with Application to Adaptive Control," Proceedings of the 47th IEEE Conference on Decision and Control, 2008.

${ }^{19}$ Yip, P.-C. P., Robust and Adaptive Nonlinear Control Using Dynamic Surface Controller with Applications to Intelligent Vehicle Highway Systems, Ph.D. thesis, University of California at Berkeley, 1997.

${ }^{20}$ Farrell, J., Polycarpou, M., and Sharma, M., "Adaptive Backstepping with Magnitude, Rate, and Bandwidth Constraints: Aircraft Longitude Control," American Control Conference, American Control Conference Council, Evanston, IL, 2003, pp. 3898-3903.

${ }^{21}$ Astolfi, A. and Ortega, R., "Immersion and Invariance: A New Tool for Stabilization and Adaptive Control of Nonlinear Systems," IEEE Transactions on Automatic COntrol, Vol. 48, No. 4, 2003, pp. 590-606.

${ }^{22}$ Astolfi, A., Karagiannis, D., and Ortega, R., Nonlinear and Adaptive Control with Applications, Springer-Verlag, 2008.

${ }^{23}$ Khalil, H. K., Nonlinear Systems, Prentice Hall, 3rd ed., 2002.

${ }^{24}$ Praly, L., "Asymptotic Stabilization via Output Feedback for Lower Triangular Systems with Output Dependent Incremental Rate," IEEE Transactions on Automatic Control, Vol. 48, 2003, pp. 1103-1108.

${ }^{25}$ Swaroop, D., Gerdes, J. C., Yip, P. P., and Hedrick, J. K., "Dynamic Surface Control of Nonlinear Systems," Proceedings of the American Control Conference, 1997.

${ }^{26}$ Page, A. B. and Steinberg, M. L., "Effects of Control Allocation Algorithms on a Nonlinear Adaptive Design," AIAA99-4282, 1999, pp. 1664-1674.

${ }^{27}$ Enns, D. F., "Control Allocation Approaches," AIAA Guidance, Navigation, and Control Conference and Exhibit, Aug. 1998.

${ }^{28}$ Forssell, L. and Nilsson, U., "ADMIRE - The Aero-Data Model in a Research Environment," Tech. rep., FOI, 2005.

\section{Appendix}

Table 2. Aircraft model parameters for flight condition 1.

\begin{tabular}{cccccc}
\hline \hline$l_{\beta}=-11.04$ & $l_{q}=0$ & $l_{r}=0.4164$ & $l_{\beta \alpha}=-19.72$ & $l_{r \alpha}=4.709$ & $l_{p}=-1.4096$ \\
$z_{\alpha}=-0.6257$ & $y_{\beta}=-0.1244$ & $m_{\alpha}=-5.432$ & $m_{\dot{\alpha}}=-0.1258$ & $m_{q}=-0.3373$ & $n_{\beta}=2.558$ \\
$n_{r}=-0.1122$ & $n_{p}=-0.0328$ & $n_{p \alpha}=-0.0026$ & $n_{q}=0$ & $l_{\delta_{e l}}=6.3176$ & $l_{\delta_{e r}}=-6.3176$ \\
$l_{\delta_{a l}}=7.9354$ & $l_{\delta_{a r}}=-7.9354$ & $l_{\delta_{r}}=1.8930$ & $i_{1}=0.7966$ & $i_{2}=0.9595$ & $i_{3}=0.6914$ \\
$m_{\delta_{e l}}=-4.5176$ & $m_{\delta_{e r}}=-4.5176$ & $m_{\delta_{a l}}=-0.8368$ & $m_{\delta_{a r}}=-0.8368$ & $m_{\delta_{l e f}}=-1.2320$ & $m_{\delta_{\text {tef }}}=0.9893$ \\
$m_{\delta_{r}}=0$ & $n_{\delta_{e l}}=0.2814$ & $n_{\delta_{e r}}=-0.2814$ & $n_{\delta_{a l}}=-0.0698$ & $n_{\delta_{a r}}=0.0698$ & $n_{\delta_{r}}=-1.7422$ \\
$V=696 \mathrm{ft} / \mathrm{s}$ & $g_{0}=32.0 \mathrm{ft} / \mathrm{s}^{2}$ & $\alpha_{0}=0.0681 \mathrm{rad}$ & $\theta_{0}=0.0681 \mathrm{rad}$ & & \\
\hline \hline
\end{tabular}

Table 3. Aircraft model parameters for flight condition 2.

\begin{tabular}{cccccc}
\hline \hline$l_{\beta}=-7.0104$ & $l_{q}=0$ & $l_{r}=0.3529$ & $l_{\beta \alpha}=-16.4015$ & $l_{r \alpha}=1.0461$ & $l_{p}=-0.7331$ \\
$z_{\alpha}=-0.2876$ & $y_{\beta}=-0.0700$ & $m_{\alpha}=-1.4592$ & $m_{\dot{\alpha}}=-0.0177$ & $m_{q}=-0.1286$ & $n_{\beta}=1.3612$ \\
$n_{r}=-0.0619$ & $n_{p}=-0.0177$ & $n_{p \alpha}=-0.0696$ & $n_{q}=0$ & $l_{\delta_{e l}}=2.7203$ & $l_{\delta_{e r}}=-2.7203$ \\
$l_{\delta_{a l}}=4.2438$ & $l_{\delta_{a r}}=-4.2438$ & $l_{\delta_{r}}=0.8920$ & $i_{1}=0.7966$ & $i_{2}=0.9595$ & $i_{3}=0.6914$ \\
$m_{\delta_{e l}}=-1.9782$ & $m_{\delta_{e r}}=-1.9782$ & $m_{\delta_{a l}}=-0.3183$ & $m_{\delta_{a r}}=-0.3183$ & $m_{\delta_{l e f}}=-0.4048$ & $m_{\delta_{t e f}}=0.3034$ \\
$m_{\delta_{r}}=0$ & $n_{\delta_{e l}}=0.1262$ & $n_{\delta_{e r}}=-0.1262$ & $n_{\delta_{a l}}=-0.0963$ & $n_{\delta_{a r}}=0.0963$ & $n_{\delta_{r}}=-0.8018$ \\
$V=581 \mathrm{ft} / \mathrm{s}$ & $g_{0}=32.0 \mathrm{ft} / \mathrm{s}^{2}$ & $\alpha_{0}=0.1447 \mathrm{rad}$ & $\theta_{0}=0.1447 \mathrm{rad}$ & & \\
\hline \hline
\end{tabular}

16 of 18 

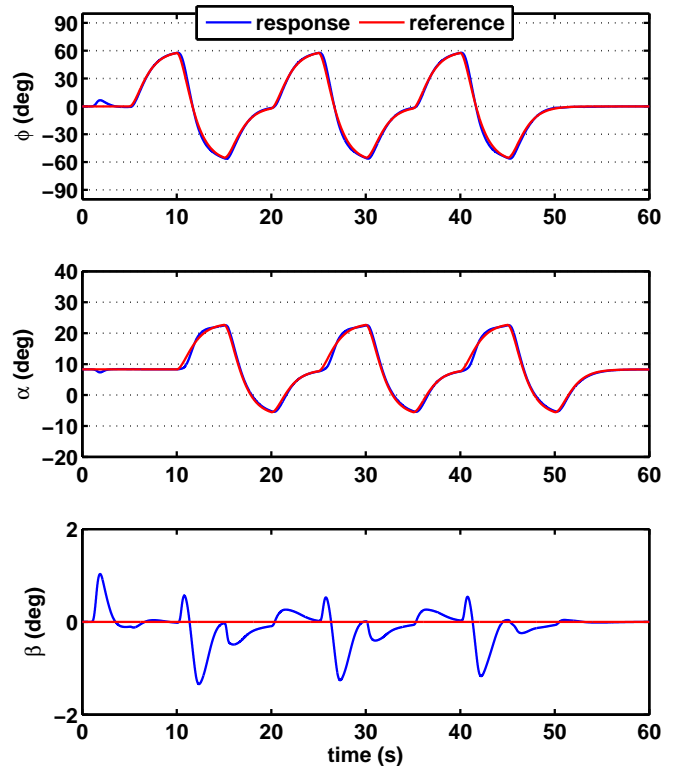

(a) Reference tracking
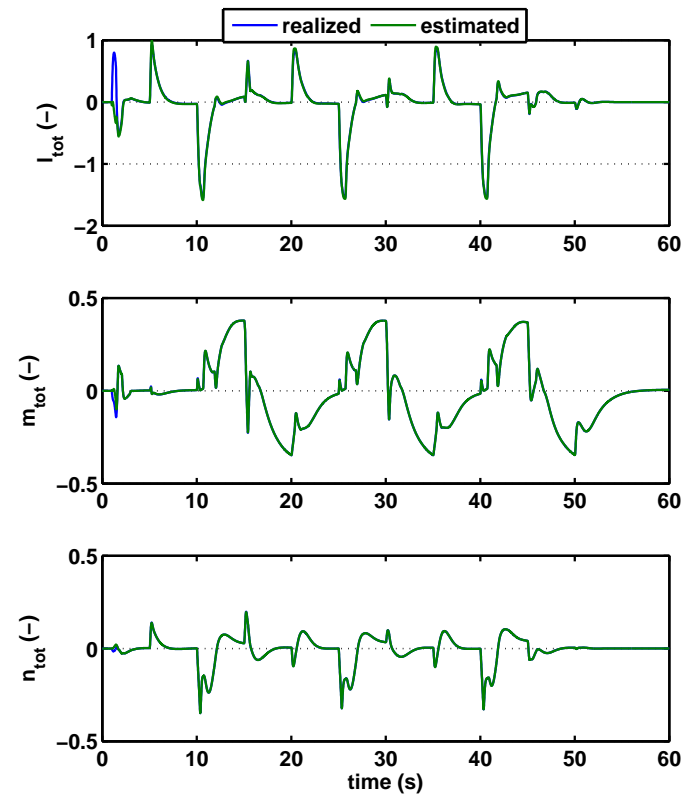

(c) Control moment
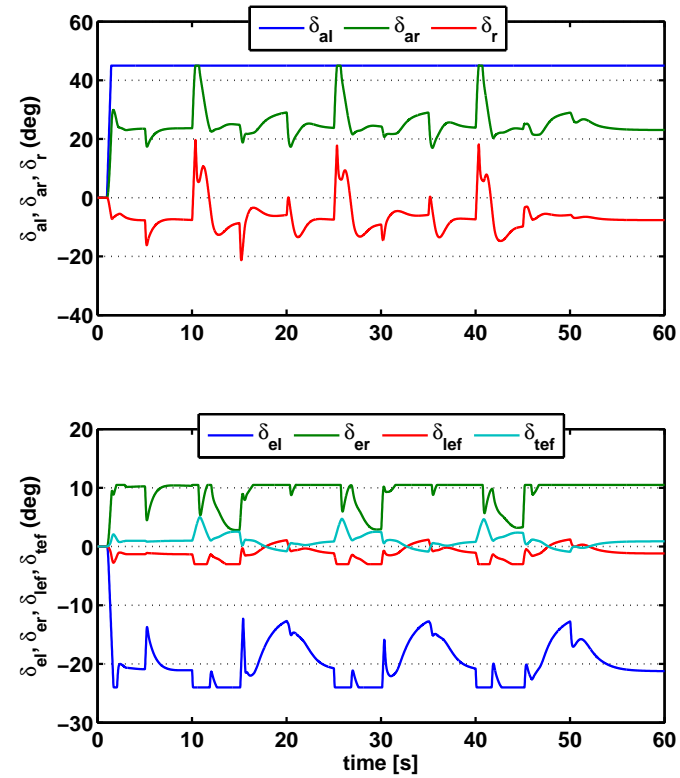

(b) Surface deflections
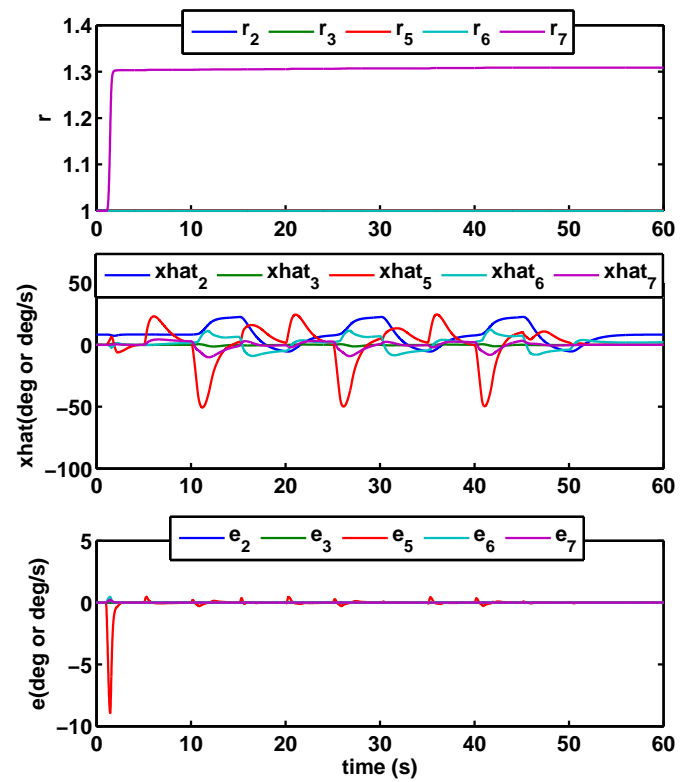

(d) Estimator Parameters

Figure 4. Simulation results at flight condition 2 for the modular adaptive controller with I\&I estimator where the aircraft experiences a hard-over of the left aileron to 45 degrees after 1 second. 

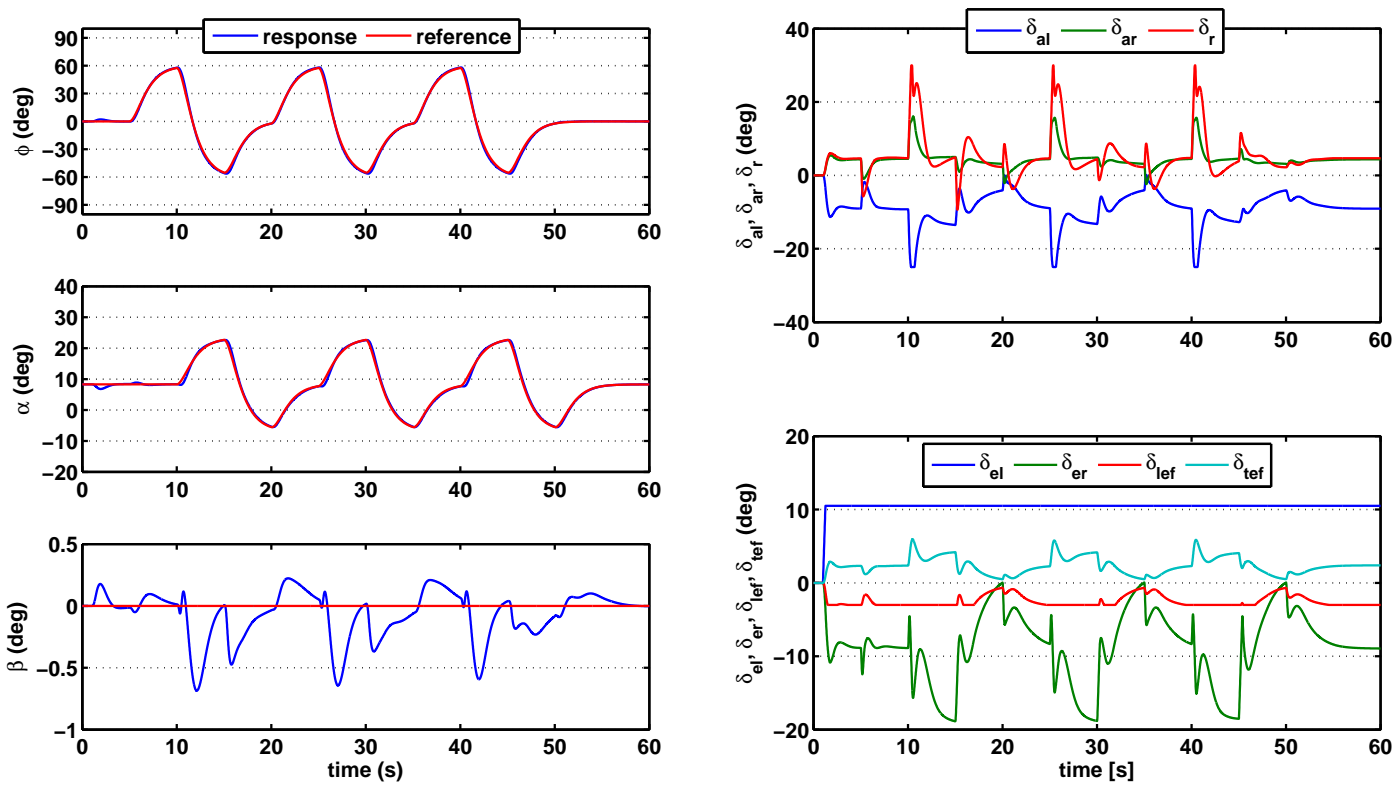

(a) Reference tracking

(b) Surface deflections
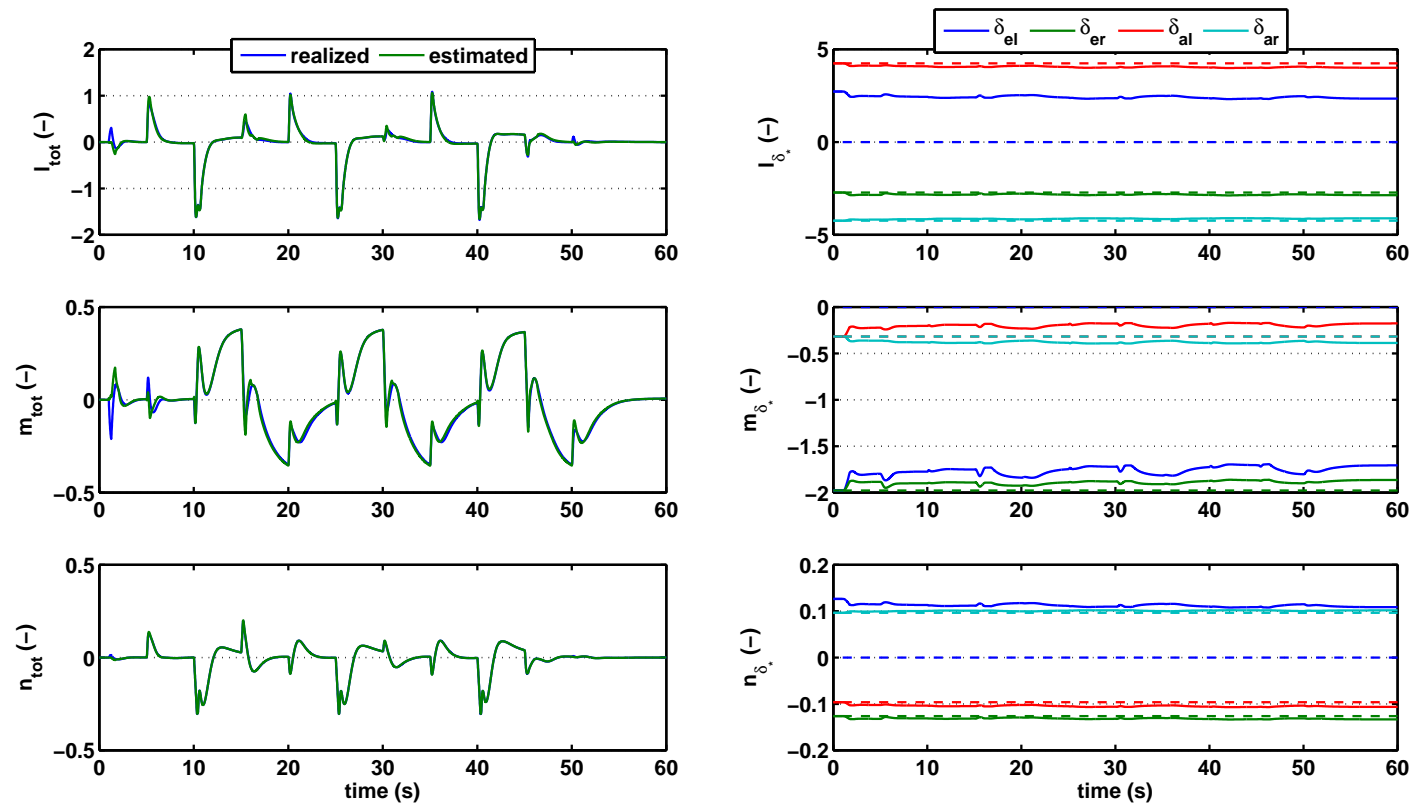

(c) Control moment

(d) Parameter estimation

Figure 5. Simulation results at flight condition 2 for the modular adaptive controller with I\&I estimator where the aircraft experiences a hard-over of the left horizontal stabilizer to 10.5 degrees after 1 second. 